\title{
HERRAMIENTA PARA EVALUAR LA GESTIÓN DE RESIDUOS HOSPITALARIOS
}

\section{TOOL TO EVALUATE THE HOSPITAL WASTE MANAGEMENT}

\author{
Luis Carlos Riofrío Cortés' ${ }^{1}$ Janneth Torres Agredo²
}

Fecha de recepción: 26 de Noviembre de 2015

Fecha de aprobación: 18 de Marzo de 2016

Referencia: L. C. Riofrío Cortés, J. Torres Agredo (2016). Herramienta para evaluar la gestión de residuos hospitalarios. Ciencia e Ingeniería Neogranadina, 26 (1), pp. 41-56, D0l: http://dx.doi.org/10.18359/rcin.1671

\section{RESUMEN}

En esta investigación se evaluó la gestión de los residuos hospitalarios en una clínica privada de tercer nivel de complejidad de la ciudad de Cali, mediante el uso de una herramienta ponderada por un panel de expertos, basada en las normas colombianas y las recomendaciones de la Organización Mundial de la Salud para la gestión de los residuos hospitalarios. La herramienta evaluó la producción de residuos, la operatividad, capacitación y cumplimiento de compromisos de los involucrados en la gestión. Se diagnosticó previamente la gestión de los residuos en la clínica, se diseñó una herramienta en Excel, y se aplicó mensualmente entre los meses de junio y septiembre de 2013. Los resultados mensuales de la medición facilitaron la identificación de las fallas en la gestión y orientaron la toma de decisiones a los responsables, lo que permitió incrementar la fracción de residuos reciclables en 3\%, reducir los residuos comunes en $2,4 \%$ y los peligrosos en $0,6 \%$. Se redujo la producción de residuos en la Unidad de Cuidados Intensivos, de $3,87 \mathrm{~kg} / \mathrm{cama} / \mathrm{dí}$ en junio, a 3,5 kg/cama/día en septiembre, y en hospitalización de 1,33kg/ cama/día a 1,25 kg/cama/día. La evaluación mensual de la gestión de estos residuos con el uso de la herramienta, permitió la identificación puntual de las fallas en la producción de residuos, el componente operativo, el programa de capacitaciones y el cumplimiento de compromisos por parte de los responsables de la gestión, lo cual entregó información valiosa para apoyar la toma de decisiones en busca de mejorar su desempeño.

1. Bioingeniero, Especialista en Ingeniería Sanitaria y Ambiental, Maestría en Ingeniería Ambiental, Universidad Nacional de Colombia, Director de proyectos Biota Ingeniería S.A.S., Cali, Colombia, Icriofrioc@unal.edu.co

2. Ing. Materiales, PhD., Profesora asociada, Grupo de investigación Materiales y Medio Ambiente, GIMMA, Universidad Nacional de Colombia, Palmira, Colombia, jtorresa@unal.edu.co 
Palabras clave: residuos hospitalarios, gestión de residuos hospitalarios, evaluación de la gestión de residuos hospitalarios, herramienta para evaluar gestión de residuos hospitalarios.

\section{ABSTRACT}

In this research, the management of hospital waste was assessed in a private hospital in third level of complexity of the Cali city, using a weighted tool by a panel of experts, based on colombian regulations and the recommendations of the World Health Organization for the management of hospital waste. The tool evaluated the production of waste, operational, training and compliance with commitments of those involved in management. Waste management in the clinic previously diagnosed an Excel tool was designed and applied on a monthly basis between june and september 2013. The monthly measurement results facilitated the identification of failures in the management and guided making responsible decisions, allowing increasing the fraction of recyclable waste by $3 \%$, reduce common waste by $2.4 \%$ and $0.6 \%$ dangerous. Waste production in Intensive Care Unit, $3.87 \mathrm{~kg} /$ bed/day in june to $3.5 \mathrm{~kg} / \mathrm{bed} /$ day in september and hospitalization of $1.33 \mathrm{~kg} / \mathrm{bed} /$ day decreased to $1.25 \mathrm{~kg} / \mathrm{bed} /$ day. The monthly assessment of the management of this waste using the tool, allowing the timely identification of faults in the production of waste, the operational component, the training program and the fulfillment of commitments by the managers, which He gave valuable information to support decision-making in seeking to improve their performance.

Keywords: hospital waste, hospital waste management, management evaluation hospital waste, tool to assess hospital waste management.

\section{INTRODUCCIÓN}

Los residuos hospitalarios representan una problemática de salud pública por su crecimiento actual y potencial de afectar la salud y el ambiente, según lo reportado por Da Silva en Brasil [1]. Según la Organización Mundial de la Salud (OMS) [2], los residuos hospitalarios causaron, en el año 2000 en el mundo, 21 millones de casos de hepatitis $\mathrm{B}$, dos millones de casos de hepatitis C y 260 mil infecciones de $\mathrm{VIH}$.

En los últimos años se han realizado esfuerzos por crear mecanismos más eficientes para su gestión, marcos legislativos, que definan los residuos hospitalarios y permitan la unificación de criterios de manejo [3]. Sin embargo, la revisión de literatura muestra que las definiciones establecidas por estos distan mucho unas de otras y hacen que sea difícil tan solo comparar las cifras publicadas [4]

Hasta el momento se han propuesto y aplicado modelos para la gestión de los residuos hospitalarios, que proponen intervenciones en la separación en las fuentes generadoras, la recolección, almacenamiento y disposición final, pero que no incluyen metodologías para la evaluación frecuente de la gestión de los residuos dentro de los centros médicos [5]. Esta situación ha dificultado el hallazgo de información y la comparación de los 
componentes metodológicos y resultados finales.

Las variaciones de cantidad y composición de los residuos está asociada, según diversos autores, al desarrollo económico del país, al ingreso per cápita, las condiciones culturales de la población, el tipo de servicios que ofrece cada centro médico y el nivel de complejidad, entre otras variables, por lo que resulta de gran utilidad disponer de modelos que permitan calificar la gestión de los residuos hospitalarios [6].

En esta investigación se propuso crear una herramienta que permitiera la evaluación frecuente de la gestión de los residuos dentro de las instituciones de salud, de manera que con los resultados obtenidos se apoyara la toma de decisiones, y con ello se lograra reducir la cantidad de residuos generados, mejorar la separación en las fuentes generadoras, disminuir los costos económicos y los impactos sobre la salud y el ambiente, lo que es consistente con lo reportado en Egipto [7]. La herramienta se diseñó con base en las normas colombianas para el manejo de residuos hospitalarios y las recomendaciones de la Organización Mundial de la Salud. Los componentes, indicadores y estándares de esta, seponderaron con un panel de expertos en gestión de residuos hospitalarios, académicos investigadores del tema, funcionarios de entes estatales de control ambiental y sanitario, y profesionales de áreas de salud con experiencia específica.

La aplicación de la herramienta se limitó a una sola clínica de nivel III de complejidad de la ciudad de Cali, con la finalidad de tener un mejor control del proceso, dado que no se encontró información de estudios similares con pautas para el desarrollo del proyecto. La complejidad de los servicios que ofrecía, el número de camas de hospitalización, el volumen de pacientes atendidos y la heterogeneidad de los residuos fueron otras de las razones para elegirla. La implementación de la herramienta para evaluar la gestión de los residuos permitió identificar tempranamente las fallas en la gestión y orientar la toma de decisiones por parte de los responsables de la gestión de los residuos para la elección y aplicación de las intervenciones.

\section{MATERIALES Y MÉTODOS}

La investigación se realizó entre los meses de abril y septiembre de 2013. Los dos primeros meses se orientaron a la socialización de la información, diagnóstico de la gestión de residuos en la clínica, el entrenamiento del personal, la construcción de la herramienta, y los cuatro meses siguientes a su aplicación en la clínica. Los datos obtenidos se procesaron con el software estadístico para las ciencias sociales SPSS versión 20.

La metodología para el estudio consistió en cinco pasos: i) diagnóstico y revisión bibliográfica, ii) desarrollo de la herramienta, iii) aplicación de la herramienta, iv) resultados, y v) análisis y conclusiones.

La investigación se desarrolló en una clínica de tercer nivel de complejidad de la ciudad de Cali (Colombia). Esta funciona 24 horas al día, siete días a la semana, presta servicios de tercer nivel de complejidad y ofrece la atención de urgencias, cirugía, hospitalización, unidad materno-fetal, unidad de imagenología, hemodinamia, unidad de cuidados intensivos adultos (UCI), UCl neonatal, unidad oncológica, consulta externa, laboratorio clínico y una oferta total de 212 camas. 
La clínica fue elegida para desarrollar la investigación por dos razones, en primer lugar porque en la ciudad no se contaba, al momento de la investigación, con datos de producción en este tipo de instituciones, por lo cual los obtenidos serían relevantes. En segundo lugar, según el informe de residuos peligrosos del Departamento Administrativo de Gestión del Medio Ambiente (DAGMA) [8], año 2009, la clínica aportó el 4,62\% del total de los residuos hospitalarios peligrosos de la ciudad de Cali.

\subsection{Diagnóstico}

La clínica no realizaba auditorías regulares para validar la separación de los residuos en las diferentes áreas. El uso de elementos de protección personal (epp) por parte de colaboradores, las condiciones de la unidad de almacenamiento de residuos y el cumplimiento de la empresa de recolección externa tampoco se evaluaban ni registraban en forma regular. Las unidades de almacenamiento central e intermedio presentaban incumplimientos frente al Manual para la Gestión Integral de Residuos Hospitalarios y Similares (MGIRHS) [9], en algunos casos porque fueron construidas antes de la norma actual, y las dimensiones o el diseño no se ajustaban, en otros casos, por falta de señalización, condiciones de higiene deficiente y/o por fallas en el uso que hacían los operarios de aseo de esta.

Se llevó a cabo una extensa consulta bibliográfica, consistente en la revisión de artículos sobre la gestión de residuos hospitalarios, informes oficiales, normatividad colombiana relacionada con residuos hospitalarios, consulta con expertos en el tema y otras fuentes de información. La revisión permitió evidenciar las características del problema de estudio y determinar los referentes teóricos más relevantes. Se revisaron publicaciones que con anterioridad han abordado el tema de la gestión de los residuos hospitalarios en Colombia y el mundo para reforzar la estructuración del marco teórico.

\subsection{Socialización del proyecto}

El proyecto se presentó a la persona encargada de la gestión de los residuos de la clínica, a quien se explicó el objetivo y los beneficios esperados. Una vez aceptado, el proyecto se socializó con el grupo de 43 operarios de aseo y las supervisoras. La socialización se realizó mediante una presentación en la que se explicó el proyecto, los beneficios esperados y la importancia de su participación para la recolección de información.

Se realizaron jornadas de capacitación en: gestión integral de los residuos de la clínica, diligenciamiento de formatos de recolección de información, normas de bioseguridad y prevención del riesgo biológico, manejo seguro de sustancias químicas, uso de elementos de protección personal y manejo de residuos. Se emplearon metodologías de presentación magistral, métodos lúdicos (el uso de juegos de separación y manejo de residuos) y audiovisuales.

\subsection{Desarrollo de la herramienta}

La herramienta se desarrolló para calificar en términos porcentuales la gestión de los residuos dentro de una institución de salud de nivel III de complejidad. La herramienta se conformó con cuatro componentes; la Figura 1 presenta el esquema de la conformación de la herramienta. Los componentes, indicadores y estándares se definieron de acuerdo con la Resolución 1164 de 2002 de los Ministerios de Medio Ambiente y Salud [10], se ponderaron 


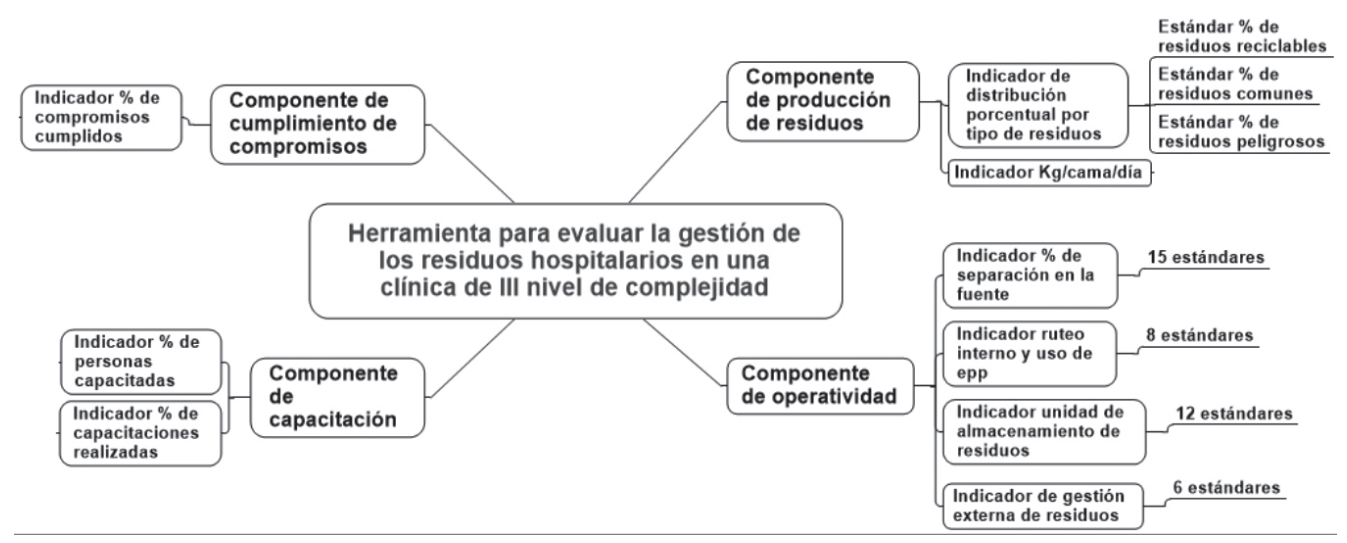

Figura 1. Esquema de herramienta para evaluar la gestión de residuos hospitalarios en una clínica de nivel III de complejidad.

Fuente: Elaboración propia, 2013

tomando como base encuestas realizadas por triplicado a un grupo de expertos en la gestión de residuos hospitalarios, según el método Delphi [11]. Los expertos entrevistados fueron académicos con experiencia en residuos hospitalarios, profesionales en áreas de ingeniería y afines, con experiencia en la gestión de residuos en centros médicos de alta complejidad, funcionarios públicos encargados de la vigilancia y el control de la gestión de residuos hospitalarios $y$, finalmente, profesionales de otras áreas con experiencia en la gestión de residuos hospitalarios en clínicas de alta complejidad.

En el componente de producción de residuos se adoptaron como indicadores: el de kg/cama/ día, que mide la producción diaria de residuos por cama y que, según Marmolejo [12], puede ser útil y confiable para estimar y monitorear la producción de residuos.

Para el monitoreo de la composición porcentual por tipo de residuos producidos, se adoptó la distribución de las categorías de residuos peligrosos, comunes y reciclables.
Esta distribución es la base para reportar la destinación de los residuos hospitalarios a las autoridades en Colombia, según el MGIRHS.

Las informaciones para alimentar el indicador de kg/cama/día y la distribución porcentual se obtuvieron del reporte de ocupación de camas generado por enfermería y el Formato de Registro de Residuos (RH1), establecido en el MGIRHS como instrumento para el registro diario de los residuos producidos en centros médicos en Colombia.

Bajo el nombre de operatividad, se agruparon las actividades relacionadas con la separación de residuos en la fuente generadora, la recolección interna y las condiciones de seguridad de los operarios de aseo, almacenamiento interno y gestión externa de los residuos. Para la recolección de la información de este componente se utilizó una lista de chequeo basada en el MGIRHS. Este permite obtener información sobre la gestión de los residuos en las diferentes áreas de la clínica, la identificación de incumplimiento de 
los estándares y orienta al responsable de la gestión de los residuos sobre las medidas a tomar para mejorar la gestión y los espacios en donde son de mayor prioridad.

El componente de capacitación reviste especial importancia en la gestión de los residuos hospitalarios. Estudios realizados por diversos autores han demostrado que la capacitación influye notablemente en la gestión de los residuos en aspectos como: la reducción de la cantidad, mejora la separación en las fuentes generadoras y reducción de la fracción peligrosa de los residuos [13]. El MGIRHS, define, además, que se debe contar con un programa de capacitaciones como parte de la gestión de residuos en centros médicos y que este debe evaluar aspectos como el porcentaje de capacitaciones realizadas y de personas capacitadas. El componente tomó como fuente de información el cronograma anual de capacitaciones y las listas de asistencia para calificar la gestión.

El cuarto componente de la herramienta se definió como el de cumplimiento de los compromisos de la gestión de residuos por parte del responsable. El indicador se tomó como el porcentaje de compromisos cumplidos. Los cuatro componentes y sus estándares se ponderaron por parte de un panel de once expertos, mediante el diligenciamiento por triplicado de una encuesta de acuerdo con el método Delphi [11]. Cada uno de los estándares se tomó con base en el MGIRHS, recomendaciones de la Organización Mundial de la Salud y la guía para la gestión de los residuos hospitalarios en centros médicos [14].

\subsubsection{Ponderación de la herramienta}

La ponderación por parte de los expertos de los indicadores kg/cama/día y de distribución porcentual de residuos, presentó igual peso porcentual de $50 \%$. El coeficiente de variación promedio fue de $21,41 \%$ lo que indica baja dispersión de los conceptos de los expertos. La Tabla 1 muestra los resultados del análisis estadístico del componente de producción de residuos.

Tabla 1. Resultados ponderación porcentual de los indicadores kg/cama/día y distribución porcentua

\begin{tabular}{|c|c|c|}
\hline $\begin{array}{c}\text { Parámetro } \\
\text { estadístico }\end{array}$ & $\begin{array}{c}\text { Kg/cama/ } \\
\text { día }\end{array}$ & $\begin{array}{c}\text { Distribución } \\
\text { porcentual } \\
\text { de la masa de } \\
\text { residuos }\end{array}$ \\
\hline Media & $50 \%$ & $50 \%$ \\
\hline Desviación estándar & 10,71 & 10,71 \\
\hline Mínimo & 35 & 20 \\
\hline Máximo & 80 & 65 \\
\hline $\begin{array}{c}\text { Nivel de confianza } \\
\text { (95,0\%) }\end{array}$ & 3,998 & 3,998 \\
\hline $\begin{array}{c}\text { Coeficiente de } \\
\text { variación }\end{array}$ & $21,34 \%$ & $21,48 \%$ \\
\hline
\end{tabular}

Fuente: Elaboración propia, 2013

La Tabla 2 presenta los resultados de la ponderación del componente de operatividad, donde la mayor ponderación fue "separación en la fuente y código de colores", con un 32,31\%, lo que indica consenso en los conceptos de los panelistas en que la separación de los residuos en la fuente generadora es la base para la gestión de los residuos hospitalarios.

El componente de capacitación consistió en dos indicadores, uno de eficiencia, que relaciona el número de capacitaciones realizadas del total programadas, y el segundo de cobertura, que relaciona el número de personas capacitadas sobre el total de personas a capacitar; estos 
Tabla 2. Ponderación componente operativo

\begin{tabular}{|c|c|c|c|c|c|c|c|}
\hline & $\begin{array}{c}\text { Media } \\
\%\end{array}$ & $\begin{array}{c}\text { Desviación } \\
\text { estándar }\end{array}$ & Rango & Mínimo & Máximo & $\begin{array}{c}\text { Nivel de } \\
\text { confianza } \\
\mathbf{9 9 , 0} \mathbf{9})\end{array}$ & $\begin{array}{c}\text { Coeficiente } \\
\text { de variación } \\
\%\end{array}$ \\
\hline $\begin{array}{c}\text { Separación en la fuente y código } \\
\text { de colores }\end{array}$ & 32,3 & 7,21 & 30 & 25 & 55 & 2,74 & 22,30 \\
\hline Ruteo interno y uso de epp. & 21,5 & 3,67 & 20 & 10 & 30 & 1,40 & 17,00 \\
\hline $\begin{array}{c}\text { Unidad técnica de } \\
\text { almacenamiento de residuos } \\
\text { (UTA) }\end{array}$ & 21,9 & 5,12 & 20 & 10 & 30 & 1,95 & 23,36 \\
\hline $\begin{array}{c}\text { Gestión externa (cumplimiento } \\
\text { de la empresa de recolección } \\
\text { externa) }\end{array}$ & 24,2 & 5,03 & 15 & 15 & 30 & 1,91 & 20,78 \\
\hline
\end{tabular}

Fuente: Elaboración propia, 2013.

se tomaron del MGIRHS [9]. La Tabla 3 presenta los resultados de la ponderación. Los expertos ponderaron la cobertura de esta, con $56,33 \%$, ligeramente superior al cumplimiento del número de capacitaciones, con 43,67\%. El coeficiente de variación del indicador de cobertura fue inferior al del número de capacitaciones realizadas, lo que indica consenso entre los expertos con respecto a la ponderación de este indicador.
El componente de cumplimiento de compromisos evaluó los del responsable de la gestión de los residuos hospitalarios. Los compromisos en la gestión de residuos se originan como resultado de las exigencias legales y como parte de los procesos necesarios para mejorar el desempeño de alguno de los componentes de la gestión de los residuos. Este se tomó como el número de compromisos cumplidos del total de compromisos adquiridos. La Ecua-

Tabla 3. Ponderación porcentual del componente de capacitación

\begin{tabular}{|c|c|c|c|c|c|c|c|}
\hline & $\begin{array}{c}\text { Media } \\
\%\end{array}$ & $\begin{array}{c}\text { Desviación } \\
\text { estándar }\end{array}$ & Rango & Mínimo & Máximo & $\begin{array}{c}\text { Nivel de } \\
\text { confianza } \\
\text { (95,0\%) }\end{array}$ & $\begin{array}{c}\text { Coeficiente } \\
\text { de } \\
\text { variación \% }\end{array}$ \\
\hline $\begin{array}{c}\text { Número de personas } \\
\text { capacitadas del total de } \\
\text { personas por capacitar }\end{array}$ & 56,3 & 11,52 & 40 & 30 & 70 & 4,30 & 20,44 \\
\hline $\begin{array}{c}\text { Número de } \\
\text { capacitaciones } \\
\text { realizadas del total de } \\
\text { programadas }\end{array}$ & 43,6 & 11,52 & 40 & 30 & 70 & 4,30 & 26,38 \\
\hline
\end{tabular}

Fuente: Elaboración propia, 2013. 
ción 1 presenta el indicador de cumplimiento de compromisos.

$$
\begin{gathered}
\% \text { de Cumplimiento } \\
\text { decompromisos }
\end{gathered}=\frac{\text { (Compromisos cumplidos) }}{\text { (Compromisos adquiridos) }} * 100
$$

La ponderación de los cuatro componentes de la herramienta mostró que el componente mejor ponderado fue el cumplimiento del responsable de la gestión de los residuos, con $27,47 \%$. Los panelistas manifestaron que la alta ponderación obedeció a que las acciones de cumplimiento del responsable de la gestión de los residuos influyen en que los otros componentes de la gestión mejoren su desempeño. La Tabla 4 presenta los resultados de la ponderación.

\subsection{Construcción de la herramienta}

Se definieron valores de metas porcentuales para los tres tipos de residuos que se generan en la clínica. Los valores se establecieron con base en los datos de producción de residuos y ocupación de camas del año 2012; los resultados fueron $25 \%$ para reciclaje, $24 \%$ para residuos comunes y $51 \%$ para residuos peligrosos, estos datos pueden ser modificados por el usuario. La Tabla 5 presenta el formato de entrada de metas y convenciones para el indicador de distribución porcentual de residuos.

La columna Verde indica el valor porcentual a partir del cual se alcanza un cumplimiento mayor o igual a $90 \%$, que se califica como excelente en la columna convenciones. La columna Amarillo, un cumplimiento mayor e igual a $80 \%$, pero menor que $90 \%$, que se califica como aceptable, y la columna Rojo, cumplimiento menor del $80 \%$, que se califica como deficiente.

En la gestión de residuos se desea que la fracción de los reciclables se incremente. En la Tabla 5 se observa queal valor 25 , definido como meta de reciclaje, se le asignó un cumplimiento

Tabla 4. Resultado de la ponderación de los cuatro componentes de la herramienta

\begin{tabular}{|c|c|c|c|c|c|c|c|}
\hline & $\begin{array}{c}\text { Media } \\
\%\end{array}$ & $\begin{array}{c}\text { Desv. } \\
\text { estándar }\end{array}$ & Rango & Mín. & Máx. & $\begin{array}{c}\text { Nivel de confianza } \\
(95,0 \%)\end{array}$ & $\begin{array}{c}\text { Coeficiente de } \\
\text { variación } \%\end{array}$ \\
\hline $\begin{array}{c}\text { Producción de } \\
\text { residuos }\end{array}$ & 22,1 & 5,25 & 20 & 12 & 32 & 1,96 & 23,67 \\
\hline $\begin{array}{c}\text { Componente de } \\
\text { operatividad de la } \\
\text { gestión. }\end{array}$ & 26,2 & 5,23 & 21 & 17 & 38 & 1,95 & 19,96 \\
\hline $\begin{array}{c}\text { Componente de } \\
\text { capacitación }\end{array}$ & 24,1 & 4,30 & 20 & 14 & 34 & 1,61 & 17,79 \\
\hline $\begin{array}{c}\text { Cumplimiento del } \\
\text { responsable de la } \\
\text { gestión }\end{array}$ & 27,4 & 6,14 & 26 & 17 & 43 & & 2,29 \\
\hline
\end{tabular}

Fuente: Elaboración propia, 2013. 
Tabla 5. Formato de entrada de metas y convenciones para el indicador de distribución porcentual de residuos

\begin{tabular}{|c|c|c|c|c|c|c|c|}
\hline $\begin{array}{c}\text { TIPO DE } \\
\text { RESIDUO }\end{array}$ & $\begin{array}{c}\text { META \% } \\
\text { DEFINIDA }\end{array}$ & VERDE & AMARILLO & ROJO & CONVENCIONES & CONDICIÓN & $\begin{array}{c}\text { \% DE } \\
\text { CUMPLIMIENTO }\end{array}$ \\
\hline RECICLAJE & 25,00 & 22,5 & 20 & 20 & EXCELENTE & $X>=$ & 90 \\
\hline COMUNES & 24,00 & 26,4 & 28,8 & 28,8 & ACEPTABLE & $X>=$ & 80 \\
\hline PELIGROSOS & 51,00 & 56,1 & 61,2 & 61,2 & DEFICIENTE & $X<$ & 80 \\
\hline
\end{tabular}

Fuente: Elaboración propia, 2013.

del $100 \%$. En la medida en que la fracción de residuos reciclables aumente más allá de 25, la calificación porcentual aumentará por encima del $100 \%$. Por ejemplo, para un valor de reciclaje del $27,5 \%$ la calificación sería del $110 \%$ $y$, por el contrario, si la fracción disminuye por debajo de 25, también disminuirá el porcentaje de cumplimiento en forma proporcional; si la recuperación de reciclables fuera de 22,5, entonces el cumplimiento porcentual de la recuperación sería del $90 \%$; si fuera de 20 , entonces el cumplimiento sería del $80 \%$ y así sucesivamente. Si el valor de recuperación disminuye, también lo hará el cumplimiento porcentual en proporción directa.

En el caso de los residuos peligrosos y comunes, se desea que las cantidades disminuyan. En la Tabla 5 se asignó a la fracción de residuos peligrosos el valor 51, como el equivalente al 100\% de cumplimiento; es decir, como la máxima fracción porcentual de residuos peligrosos que debería generar mensualmente la clínica, por ello si la fracción de residuos peligrosos supera este valor máximo establecido, el porcentaje de cumplimiento de la meta se reducirá. Como ejemplo, la Tabla 5 muestra que cuando la fracción de residuos peligrosos alcanzó la cifra de 56,1 , el valor de cumplimiento fue del $90 \%$, para el valor de 61,2 entonces el cumplimiento fue del $80 \%$ y así sucesivamente; por el contrario, si la fracción de residuos peligrosos fuese de 45,9, la calificación sería de 110\%, y a menor fracción del residuos, la calificación será mayor al 100\%. Se observa que para residuos comunes y peligrosos la relación de cantidad generada y la calificación es inversamente proporcional.

La Tabla 6 presenta las celdas para la entrada de los valores definidos como meta para el indicador de kg/cama/día en UCl y hospitalización.

Las metas para este indicador se establecieron a partir del análisis de los datos de ocupación de cama y producción de residuos del año 2012, se definieron las metas en kg/cama/ día para UCl en 3,6 y 1,28 para hospitalización. Se asignó a los valores meta una calificación del $100 \%$ y, al igual que para las metas en residuos peligrosos y comunes, la calificación es inversamente proporcional.

El ingreso de datos para los componentes de la herramienta consta de cinco pasos, en los que mensualmente se ingresan los datos recolectados y que, mediante la aplicación de las ponderaciones realizadas por los expertos, arroja los resultados porcentuales de la evaluación de cada uno de los componentes. 
Tabla 6. Formato de ingreso de datos para el indicador kg/cama/día para UCI y hospitalización

\begin{tabular}{|c|c|c|c|c|}
\hline TIPO DE RESIDUO & $\begin{array}{c}\text { META \% } \\
\text { DEFINIDA }\end{array}$ & $\begin{array}{c}\text { VERDE } \\
\text { (EXCELENTE) }\end{array}$ & $\begin{array}{c}\text { AMARILLO } \\
\text { (ACEPTABLE) }\end{array}$ & $\begin{array}{c}\text { ROJO } \\
\text { (DEFICIENTE) }\end{array}$ \\
\hline KG/CAMA/DÍA-UCI & 3,60 & 3,96 & 4,32 & 4,32 \\
\hline $\begin{array}{c}\text { KG/CAMA/DÍA } \\
\text {-HOSPITALIZACIÓN }\end{array}$ & 1,28 & 1,408 & 1,536 & 1,536 \\
\hline
\end{tabular}

Fuente: Elaboración propia. 2013.

\section{RESULTADOS Y ANÁLISIS}

En la Figura 2 se presenta la distribución porcentual de los residuos peligrosos, comunes y reciclables entre los meses de junio y septiembre del año 2013. En esta se observa que, al finalizar los cuatro meses de aplicación de la herramienta, la fracción de residuos peligrosos presentó una media de $53,2 \%$, la más alta de los tres tipos de residuos, superior al $40 \%$ reportado para Colombia [9]. Se requiere, sin embargo, actualizar la estimación de la distribución porcentual de los residuos en los diferentes niveles de complejidad en el país, para tener una base con la cual comparar. Los residuos comunes alcanzaron una media de $23,89 \%$ en el periodo y los reciclables de $22,89 \%$. Sobre estos dos últimos tipos de residuos no se encontraron datos discriminados de su participación porcentual en la literatura; sin embargo, se hace necesario contar con estos para definir las acciones tendientes a mejorar la gestión de los residuos hospitalarios.

Figura 2. Distribución porcentual de los residuos peligrosos, comunes y reciclables entre junio y septiembre de 2013

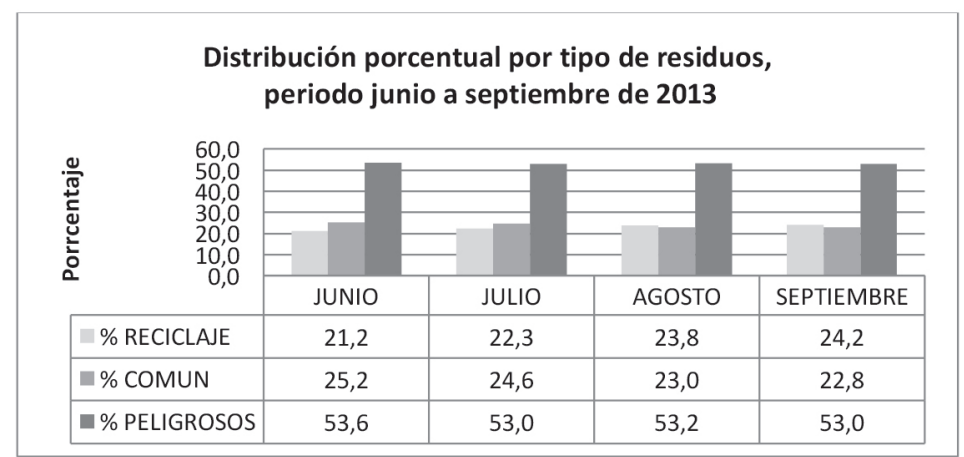

Fuente: Elaboración propia. 
La Figura 2 muestra que los residuos peligrosos se redujeron en $0,6 \%$, los comunes en $2,4 \%$, mientras que los reciclables se incrementaron en $3 \%$, lo cual indica que los residuos reciclables crecieron aexpensas de los comunes y en menor medida de los peligrosos. Lo anterior evidenció que residuos reciclables se dispusieron como comunes y peligrosos, lo que incrementa los costos de disposición final.

Durante el periodo descendió la producción de residuos en 0,37 kg/cama/día en $\mathrm{UCl}$, al pasar de $3,87 \mathrm{~kg} / \mathrm{cama} / \mathrm{dí}$ a $3,50 \mathrm{~kg} / \mathrm{cama} /$ día, superior a la cifra nacional de producción de 3,06 reportado en la Política Ambiental para la Gestión de Residuos o Desechos Peligrosos de 2005 [15]. Esta reducción en la cantidad de residuos es consistente con lo reportado por Moreira en 2013 [16], quien informa reducción en las cantidades de residuos generados en $\mathrm{kg} /$ cama/día, además de incremento de la fracción de residuos reciclables y reducción de los residuos comunes; lo anterior es consistente con lo reportado en centros médicos de alta complejidad en China [17]. Un comparativo entre el indicador de $\mathrm{kg} / \mathrm{cama} / \mathrm{dí}$ de UCl y hospitalización mostró que la producción de residuos en UCl fue, en promedio, de 2,75 veces la de hospitalización entre los meses evaluados. La Tabla 7 presenta los resultados de la evaluación del componente de producción de residuos en el periodo.

El componente operativo, por su parte, alcanzó un cumplimiento del $88,47 \%$ en junio, y finalizó en septiembre con $96,32 \%$. Se observó en el periodo un incremento del cumplimiento de $7,85 \%$. El indicador de gestión externa presentó la mayor calificación del periodo con 100\%. La separación en la fuente presentó el mayor crecimiento, al pasar de una calificación de $86,93 \%$ a $98,50 \%$, con un crecimiento del 11,57\%. La unidad dealmacenamiento presentó el segundo crecimiento, al pasar de $77,96 \%$ a $88,91 \%$, con un crecimiento del $10,95 \%$, y en tercer lugar se ubicó el ruteo interno y uso de elementos de protección personal (epp), con un crecimiento de $8,86 \%$. La Tabla 8 presenta los resultados del componente en el periodo.

El componente de capacitación alcanzó en junio una calificación del $79,18 \%$ y de $87,33 \%$ en

Tabla 7. Resultados de la evaluación del componente de producción de residuos entre junio y septiembre de 2013

\begin{tabular}{|c|c|c|c|c|}
\hline CUMPLIMIENTO DE METAS (\%) & JUNIO & JULIO & AGOSTO & SEPTIEMBRE \\
\hline Reciclaje & 84,81 & 89,39 & 95,19 & 96,90 \\
\hline Comunes & 94,97 & 97,47 & 104,26 & 105,01 \\
\hline Peligrosos & 94,92 & 95,99 & 95,64 & 96,12 \\
\hline $\begin{array}{c}\text { De cumplimiento distribución de } \\
\text { residuos por tipo }\end{array}$ & 97,75 & 94,28 & 98,36 & 99,34 \\
\hline $\begin{array}{c}\text { De cumplimiento kg/cama/día } \\
\text { De cumplimiento total componente } \\
\text { producción de residuos }\end{array}$ & 94,09 & 99,60 & 102,20 & 102,54 \\
\hline
\end{tabular}

Fuente: Elaboración propia, 2013. 
septiembre. En junio el indicador de capacitaciones realizadas obtuvo $66,67 \%$, mientras que el de personas capacitadas alcanzó 88,89\%. Lo anterior se asoció al incumplimiento de algunas actividades de capacitación programadas y, por otro lado, a las coberturas no alcanzadas. En agosto el componente alcanzó 98,74\%, la más alta calificación del periodo, jalonada por el cumplimiento de los indicadores de capacitaciones realizadas y cobertura, con 100 y $97,78 \%$, respectivamente. En septiembre el componente obtuvo una calificación de $87,33 \%$, lo que indica al responsable la necesidad de realizar seguimiento permanente al componente para asegurar resultados óptimos. La Tabla 9 presenta los resultados del componente.

El componente de cumplimiento de compromisos alcanzó, durante tres de los cuatro meses del periodo, una calificación del $100 \%$, a excepción de agosto, en donde descendió al 80\%. El resultado indica que los compromisos adquiridos por los responsables de la gestión de los residuos en la clínica se cumplieron en su gran mayoría. Esta situación indicaría un importante compromiso por parte de los responsables de la gestión de residuos en la clínica. La Tabla 10 presenta los resultados alcanzados por el componente en el periodo.

Tabla 8. Resultados del componente operativo entre junio y septiembre de 2013

\begin{tabular}{|c|c|c|c|c|}
\hline ETAPA & $\begin{array}{c}\text { JUNIO } \\
(\%)\end{array}$ & $\begin{array}{c}\text { JULIO } \\
(\%)\end{array}$ & $\begin{array}{c}\text { AGOSTO } \\
(\%)\end{array}$ & $\begin{array}{c}\text { SEPTIEMBRE } \\
(\%)\end{array}$ \\
\hline SEPARACIÓN EN LA FUENTE Y CÓDIGO DE COLORES & 86,93 & 96,81 & 94,69 & 98,50 \\
\hline $\begin{array}{c}\text { RUTEO INTERNO Y USO DE ELEMENTOS DE } \\
\text { PROTECCIÓN PERSONAL }\end{array}$ & 89,00 & 97,00 & 89,71 & 97,86 \\
\hline $\begin{array}{c}\text { UNIDAD TÉCNICA DE ALMACENAMIENTO DE } \\
\text { RESIDUOS }\end{array}$ & 77,96 & 83,51 & 85,47 & 88,91 \\
\hline GESTIÓN EXTERNA & 100 & 100 & 100 & 100 \\
\hline TOTAL & 88,47 & 94,34 & 92,47 & 96,32 \\
\hline
\end{tabular}

Fuente: Elaboración propia, 2013

Tabla 9. Resultados de evaluación de componente de capacitaciones: mes de junio.

\begin{tabular}{|c|c|c|c|c|}
\hline INDICADORES (\%) & JUNIO & JULIO & AGOSTO & SEPTIEMBRE \\
\hline CAPACITACIONES REALIZADAS & 66,67 & 75,00 & 100,00 & 87,50 \\
\hline PERSONAS CAPACITADAS & 88,89 & 90,67 & 97,78 & 87,21 \\
\hline CUMPLIMIENTO PORCENTUAL CAPACITACIÓN & 79,18 & 83,82 & 98,74 & 87,33 \\
\hline
\end{tabular}

Fuente: Elaboración propia, 2013 
La calificación total de la gestión de los residuos hospitalarios en la clínica se incrementó en el periodo junio-septiembre de 2013 en 5,89\%, al pasar de $90,56 \%$ a 96,25 . El análisis por componentes mostró que el de compromisos mantuvo una calificación de 100\% durante tres de los cuatro meses, comportamiento atribuible a la dedicación por parte de los responsables de la gestión de los residuos en la clínica y a los controles periódicos de las autoridades sanitarias a clínicas y hospitales de alta complejidad. Desde el comienzo de la aplicación de la herramienta, y a lo largo de los cuatro meses, la calificación alcanzada por la gestión de los residuos de la clínica fue superior al $90 \%$.

La Tabla 11 presenta las calificaciones alcanzadas por cada uno de los componentes de la herramienta.
Los componentes de producción de residuos, operativo y de capacitación, alcanzaron calificaciones de $100,94 \%$, $96,65 \%$ y $87,33 \%$, respectivamente. El componente de capacitación, a pesar de tener al final de periodo la más baja calificación, presentó el mayor incremento porcentual, con $8,15 \%$, seguido del componente de operatividad, con $8,12 \%$, y finalmente el de producción de residuos, con $8,11 \%$.

Con los resultados, de manera general se encontró que la fracción de residuos peligrosos fue la de mayor participación porcentual de la masa de residuos durante el periodo, con un $53 \%$, siendo esta superior al $25 \%$ reportado por la Organización Mundial de la Salud [18], y al 40\% reportado para Colombia por los Ministerios de Salud y Medio Ambiente en 2002 [9]. Adicionalmente, la fracción de residuos peligrosos fue la mejor calificada en

Tabla 10. Resultados de evaluación del componente de cumplimiento de compromisos.

\begin{tabular}{|c|c|c|c|c|}
\hline ESTÁNDAR & JUNIO & JULIO & AGOSTO & SEPTIEMBRE \\
\hline PORCENTAJE DE CUMPLIMIENTO COMPROMISOS & 100,00 & 100,00 & 80,00 & 100,00 \\
\hline
\end{tabular}

Fuente: Elaboración propia, 2013.

Tabla 11. Resultados de evaluación de los componentes de la herramienta en el periodo junio-septiembre de 2013.

\begin{tabular}{|c|c|c|c|c|}
\hline COMPONENTES (\%) & JUNIO & JULIO & AGOSTO & SEPTIEMBRE \\
\hline PRODUCCIÓN DE RESIDUOS & 92,83 & 96,94 & 100,28 & 100,94 \\
\hline COMPONENTE OPERATIVO & 88,53 & 94,77 & 92,89 & 96,56 \\
\hline COMPONENTE DE CAPACITACIÓN & 79,18 & 83,82 & 98,74 & 87,33 \\
\hline CUMPLIMIENTO DE COMPROMISOS & 100,00 & 100,00 & 80,00 & 100,00 \\
\hline CUMPLIMIENTO TOTAL HERRAMIENTA & 90,36 & 94,02 & 92,38 & 96,25 \\
\hline
\end{tabular}

Fuente: Elaboración propia, 2013 
separación en la fuente, lo que concuerda con lo reportado por [17], donde afirmaron que los residuos peligrosos presentaron el mejor cumplimiento en separación en la fuente que los residuos no peligrosos en centros médicos de alta complejidad.

Los resultados mensuales de la herramienta orientaron al responsable de la gestión de los residuos a la toma de decisiones, dado que identificaron los elementos puntuales que generaron los incumplimientos, permitiéndole priorizar y presupuestar los recursos para dichas intervenciones.

Los componentes de producción de residuos y operativo permitieron evaluar la gestión de los residuos en las fuentes generadoras, reflejados en la distribución porcentual y la cantidad de residuos en kg/cama/día, mientras que los componentes de cumplimiento de compromisos y capacitación midieron los procesos administrativos de la gestión y de sus responsables. Lo anterior muestra que la herramienta evalúa la gestión de los residuos en sus componentes más relevantes, lo que concuerda con lo reportado por la Organización Mundial de la Salud [19].

\section{CONCLUSIONES}

A partir de los resultados obtenidos se puede concluir que:

La identificación temprana de las falencias en separación en la fuente con la herramienta permitió direccionar las intervenciones y mejorar los resultados, con la consecuente reducción de costos por disposición final.

La producción de residuos hospitalarios en la clínica evaluada descendió a 3,5kg/cama/ día en la unidad de cuidados intensivos $(0,37$ $\mathrm{kg} /$ cama/día) y a 1,25 (0,08 kg/cama/día) en hospitalización durante el periodo.

Los componentes de producción de residuos y operativo permitieron evaluar la gestión de los residuos en las fuentes generadoras, reflejados en la distribución porcentual y la cantidad de residuos en kg/cama/día, mientras que los componentes de cumplimiento de compromisos y capacitación midieron los procesos administrativos de la gestión y de sus responsables.

Los resultados mensuales de la herramienta orientaron al responsable de la gestión de los residuos a la toma de decisiones oportunas, dado que identificaron los elementos puntuales que generaron los incumplimientos mes a mes, permitiéndole priorizar y presupuestar los recursos para dichas intervenciones.

Vale la pena mencionar que la herramienta desarrollada, puede ser aplicada en otros centros médicos de diferentes niveles de complejidad; lo que permitiría identificar falencias y puntos fuertes de la gestión de los residuos en dichas entidades y orientar decisiones más efectivas.

\section{BIBLIOGRAFÍA}

[1] Da Silva, C. E., Hoppe, A. E., Ravanello, M. M., Mello, N. (2005). Medical wastes management in the south of Brazil. Waste Management, 25 (6), pp. 600-5.

[2] World Health Organization. (2007). WHO core principles for achieving safe and sustainable management of health-care waste. Geneva. 
[3] Díaz, L. F., Eggerth, L.L., Enkhtsetseg, S.H., Savage, G.M. (2008). Characteristics of healthcare wastes. Waste Management, 28(7), pp. 1219-26.

[4] Prem Ananth, A., Prashanthini, V., Visvanathan C. (2010). Healthcare waste management in Asia. Waste Management, 30(1), pp. 154-61.

[5] Bulucea, C. A. V., Bulucea, A. V., Popescu, M. C., Patrascu, A. F. (2008). Assessment of Biomedical Waste Situation in Hospitals of Dolj District. International Journal of Biology And Biomedical Engineering, 2(1), pp 19-28.

[6] Cabello, R., Sauma, E. (2007). Un modelo de generación de residuos hospitalarios para la región metropolitana de Chile. Revista de la Ingeniería Industrial, 1(1), pp. 1-9.

[7] Abd El-Salam, M. M. (2010). Hospital waste management in El-Beheira Governorate, Egypt. Journal of Environmental Management, 91(3), pp. 618-629.

[8] Departamento Administrativo de Gestión del Medio Ambiente. (2012). Informe consolidado sobre el registro de generadores de residuos o desechos peligrosos - Periodo de Balance Declarado año 2009. Santiago de Cali.

[9] Ministerio de Salud. Ministerio del Medio Ambiente. (2002). Manual de Procedimientos para la Gestión Integral de los Residuos Hospitalarios y Similares. Bogotá.

[10] Ministerio de Salud. Ministerio del Medio Ambiente. (2002). Resolución 1164 de 2002. Bogotá.
[11] Linstone, H. A., Turoff, M. (2002). The Delphi Method. Reading. Addison-Wesley Publishing Company Inc., pp. 1-168.

[12] Marmolejo, L. F., Madera, C. A., Torres, P. (2010). Gestión de los residuos sólidos en hospitales locales del norte del Valle del Cauca, Colombia. Revista Facultad Nacional de Salud Pública, 28(1), pp. 5663.

[13] Mosquera M., Andrés, Prado, M. J., Rodríguez-Caravaca, G., Latasa, P., Mosquera, E. G. (2014). Evaluation of an education and training intervention to reduce health care waste in a tertiary hospital in Spain. American Journal of Infectin Control, 42 (8), pp. 894-897.

[14] Centro Panamericano de Ingeniería Sanitaria y Ciencias para el Ambiente. (1995). Guía para el Manejo Interno de Residuos Sólidos en Centros de Atención de Salud. (2 Ed) Lima: Cepis/OPS.

[15] Ministerio de Ambiente Vivienda y Desarrollo Territorial. (2005). Política ambiental para la gestión integral de residuos o desechos peligrosos. Bogotá. Oficina de Prensa MAVDT.

[16] Maniero, A. M., Risso, W. M. (2013). Evaluation of Medical Waste Management applied to a Small Capacity Healthcare Unit in Brazil. Facultad de Salud Pública de Universidad de São Paulo.

[17] Ruoyan, G., Lingzhong X., Huijuan, L., Chengchao, Z., Jiangjiang, $H_{\text {., Yoshihisa, }}$ S., Wei, T., Chushi, K. (2010). Investigation of health care waste management in Binzhou District, China. Waste Management, 30(2), pp. 246-250. 
[18] World Health Organization. (1999). Safe management of wastes from health care activities. Geneva. World Health Organization.

[19] World Health Organization. (2007). Core principles for achieving safe and sustainable management of healthcare waste. Safe health-care waste management, pp.1-2. Available at: www. healthcarewaste.org. 